\title{
Testing the distance-duality relation to probe the morphology of galaxy clusters
}

\author{
Xiao-Lei Meng ${ }^{1,2}$ \\ ${ }^{1}$ Department of Astronomy, Beijing Normal University, Beijing 100875, China \\ email: mlwx@mail.bnu.edu.cn \\ ${ }^{2}$ National Astronomical Observatories, Chinese Academy of Sciences, Beijing 100012, China
}

\begin{abstract}
Aiming at comparing different morphological models of galaxy clusters, we use two new methods to construct a cosmological-model-independent test of the distance-duality (DD) relation. The luminosity distances come from the UNION2 compilation of Type Ia supernovae. The angular-diameter distances are given by the two cluster models of De Filippis et al. and Bonamente $e t$ al. The advantage of our new methods is that they can lead to reduced statistical errors. The morphologies of the cluster models are mainly focused on a comparison between the elliptical and spherical $\beta$ models. The spherical $\beta$ model is divided into two groups, in terms of different reduction methods of angular-diameter distances, i.e. the conservative and corrected spherical $\beta$ models. Our results show that the DD relation is consistent with the elliptical $\beta$ model at the $1 \sigma$ confidence level (CL) for both methods, whereas for almost all spherical $\beta$ model parameterizations the DD relation can only be accommodated at the $3 \sigma \mathrm{CL}$, particularly for the conservative spherical $\beta$ model. To minimize systematic uncertainties, we also apply the test to the overlap sample, i.e. to the same set of clusters modeled by both De Filippis et al. and Bonamente et al. The DD relation is compatible with the elliptically modeled overlap sample at the $1 \sigma \mathrm{CL}$; however, for most parameterizations the DD relation cannot be accommodated even at the $3 \sigma$ CL by any of the two spherical $\beta$ models. Therefore, it is reasonable that the marked triaxial ellipsoidal model is a better geometric hypothesis describing the structure of galaxy clusters than the spherical $\beta$ model if the DD relation is valid for cosmological observations.
\end{abstract}

Keywords. cosmic background radiation, cosmology: observations, distance scale, galaxies: clusters: general, X-rays: galaxies: clusters 